\title{
Repositioning Azelnidipine as a Dual Inhibitor Targeting CD47/SIRP $\alpha$ and TIGIT/PVR Pathways for Cancer Immuno-Therapy
}

\author{
Xiuman Zhou ${ }^{1}$, Ling Jiao ${ }^{2}$, Yuzhen Qian ${ }^{2}$, Qingyu Dong ${ }^{2}$, Yixuan Sun ${ }^{2}$, Wei V. Zheng ${ }^{1}$, Wenshan Zhao ${ }^{2}$, \\ Wenjie Zhai ${ }^{2}$, Lu Qiu ${ }^{2}$, Yahong Wu ${ }^{2} \mathbb{D}$, Hongfei Wang ${ }^{2, *}$, Yanfeng Gao ${ }^{3, *}$ and Junhui Chen ${ }^{1, *}$ \\ 1 Intervention and Cell Therapy Center, Peking University Shenzhen Hospital, Shenzhen Peking \\ University-The Hong Kong University of Science and Technology Medical Center, Shenzhen 518035, China; \\ H027@pkuszh.com (X.Z.); zhengw2013@yeah.net (W.V.Z.) \\ 2 School of Life Sciences, Zhengzhou University, Zhengzhou 450001, China; jisunny@gs.zzu.edu.cn (L.J.); \\ qyz_2021@gs.zzu.edu.cn (Y.Q.); 202022162012917@gs.zzu.edu.cn (Q.D.); sunyixuan@gs.zzu.edu.cn (Y.S.); \\ zhaowsh07@zzu.edu.cn (W.Z.); wjzhai@zzu.edu.cn (W.Z.); qiulu@zzu.edu.cn (L.Q.); \\ yahongwu@zzu.edu.cn (Y.W.) \\ 3 School of Pharmaceutical Sciences (Shenzhen), Sun Yat-sen University, Shenzhen 518107, China \\ * Correspondence: whf61300@yeah.net (H.W.); gaoyf29@mail.sysu.edu.cn (Y.G.); 1232@pkuszh.com (J.C.)
}

check for updates

Citation: Zhou, X.; Jiao, L.; Qian, Y.; Dong, Q.; Sun, Y.; Zheng, W.V.; Zhao, W.; Zhai, W.; Qiu, L.; Wu, Y.; et al. Repositioning Azelnidipine as a Dual Inhibitor Targeting CD47/SIRP $\alpha$ and TIGIT/PVR Pathways for Cancer Immuno-Therapy. Biomolecules 2021, 11, 706. https://doi.org/10.3390/ biom 11050706

Academic Editor: Stephan M. Huber

Received: 14 March 2021

Accepted: 7 May 2021

Published: 10 May 2021

Publisher's Note: MDPI stays neutral with regard to jurisdictional claims in published maps and institutional affiliations.

Copyright: (c) 2021 by the authors. Licensee MDPI, Basel, Switzerland. This article is an open access article distributed under the terms and conditions of the Creative Commons Attribution (CC BY) license (https:/ / creativecommons.org/licenses/by/ $4.0 /)$.

\begin{abstract}
Strategies boosting both innate and adaptive immunity have great application prospects in cancer immunotherapy. Antibodies dual blocking the innate checkpoint CD47 and adaptive checkpoint PD-L1 or TIGIT could achieve durable anti-tumor effects. However, a small molecule dual blockade of CD47/SIRP $\alpha$ and TIGIT/PVR pathways has not been investigated. Here, an elevated expression of CD47 and PVR was observed in tumor tissues and cell lines analyzed with the GEO datasets and by flow cytometry, respectively. Compounds approved by the FDA were screened with the software MOE by docking to the potential binding pockets of SIRP $\alpha$ and PVR identified with the corresponding structural analysis. The candidate compounds were screened by blocking and MST binding assays. Azelnidipine was found to dual block CD47/SIRP $\alpha$ and TIGIT/PVR pathways by co-targeting SIRP $\alpha$ and PVR. In vitro, azelnidipine could enhance the macrophage phagocytosis when co-cultured with tumor cells. In vivo, azelnidipine alone or combined with irradiation could significantly inhibit the growth of MC38 tumors. Azelnidipine also significantly inhibits the growth of CT26 tumors, by enhancing the infiltration and function of $\mathrm{CD} 8^{+} \mathrm{T}$ cell in tumor and systematic immune response in the tumor-draining lymph node and spleen in a CD8 ${ }^{+} \mathrm{T}$ cell dependent manner. Our research suggests that the anti-hypertensive drug azelnidipine could be repositioned for cancer immunotherapy.
\end{abstract}

Keywords: CD47/SIRP $\alpha$; TIGIT/PVR; drug-repositioning; small molecule inhibitor; azelnidipine; cancer immunotherapy

\section{Introduction}

Cancer immunotherapy represented by the blockade of immune checkpoint PD-1/PD$\mathrm{L} 1$ has gained substantial progress. The anti-PD-1/PD-L1 resistance resulting from the upregulation of immune checkpoints suggests the importance and urgency of developing new targets [1]. A combinational blockade of different targets with non-redundant functions may achieve better clinical benefits. A novel immune checkpoint $\mathrm{T}$ cell immunoglobulin and immunoreceptor tyrosine-based inhibitory motif (TIGIT), which always co-expresses with PD-1 on NK and T cells and symbolizes a more exhausted status, plays pivotal roles in the adaptive anti-tumor immunity by ligation with its major ligand poliovirus receptor (PVR) [2,3]. PVR was initially identified as the receptor for the human poliovirus, or as an adhesion-related molecule that mediates tumor invasion. Increasing attention has been paid to PVR in cancer immunotherapy since the verification of its role 
as the shared ligand for the immune checkpoint TIGIT and CD96 [4-7]. The elevated expression of PVR facilitates the immune escape of tumor cells including melanoma, head and neck squamous cell carcinoma, and colorectal cancers [8-10]. The immunosuppressive role of TIGIT on NK and T cells depends on its interaction with PVR. A blockade of receptor-ligand interaction by targeting TIGIT or PVR could reverse the exhaustion of NK and $\mathrm{CD} 8^{+} \mathrm{T}$ cells and enhance the anti-tumor immunity [3,11,12].

An important reason affecting the efficacy of immunotherapy is the infiltration of $\mathrm{CD} 8^{+}$ T cells [13]. Although targeting various immune checkpoints alone or in combination could maximize the activation of $\mathrm{T}$ cells, the response to immune checkpoint blockade therapy is extremely low in the tumors with rare $\mathrm{T}$ cell infiltration. As the important innate immune cells and one of the most abundant cells in the tumor microenvironment, macrophages play an important role in adaptive immunity and anti-tumor response [14,15]. Macrophages could maintain the tissue homeostasis by recognizing and engulfing the foreign, damaged, or dying cells, without attacking the normal cells. The precise phagocytosis mechanism of macrophages was modulated by the balance of the "eat me" and "do not eat me" signals. Normal cells express CD47 to transmit an anti-phagocytosis signal by interacting with the signal regulatory protein alpha $(\mathrm{SIRP} \alpha)$ on macrophages to avoid clearance. However, tumor cells could evade the immune surveillance via utilizing the elevated expression of CD47. A blockade of CD47/SIRP $\alpha$ could reactivate the phagocytosis of macrophages and enhance the anti-tumor immunity.

Simultaneously stimulating adaptive immunity and innate immunity has been proved to be a promising strategy to obtain maximum and durable anti-tumor immune response [16] A combination of SIRP $\alpha$ and PD-1 blockade could result in durable anti-tumor immunity by facilitating monocyte activation, dendritic cell activation, and $\mathrm{T}$ cell effector functions [17]. Dual targeting CD47 and PD-L1 by fusion protein or bispecific antibody enables effective targeting to tumor cells than non-tumor cells, increased DNA sensing, DC crosspresentation, and anti-tumor $\mathrm{T}$ cell response $[18,19]$. The bispecific antibody dual targeting CD47 and TIGIT has also been designed for cancer immunotherapy (WO2020259535). However, small molecule inhibitors that dual block the CD47/ SIRP $\alpha$ and TIGIT/PVR pathways have not been discovered.

Repositioning existing drugs for new indications has increasingly become a smart strategy for drug discovery, with definite safety and pharmacokinetic data to potentially reduce the overall costs and shorten the development timelines [20,21]. With a systematic analysis of numerous large scale data or combined with various computational approaches, many drugs has been discovered for new applications, especially the drugs approved by the FDA [22,23]. Metformin, the first-line oral medication for type 2 diabetes (T2D), has been reported to be a classical example of drug-repositioning in cancer immunotherapy by exerting anti-inflammatory effect, and enhancing the $\mathrm{T}$ cell immunity through reducing the expression of the immune checkpoint PD-L1, CD73, or participating in the metabolic reprogramming, and so on [24-26]. An increasing number of drugs have been reported to be repositioned for cancer immunotherapy [12,27].

Here, we analyzed the expression of CD47 and PVR from the GEO database and tested the existence on the tumor cell lines by flow cytometry. Based on the structure of CD47/SIRP $\alpha$ and TIGIT/PVR, the small molecules from the FDA-approved drug library with dual targeting effects on SIRP $\alpha$ and PVR were screened by docking-based virtual screening methods. The candidate inhibitors were further verified by the blocking assays. Azelnidipine was found to simultaneously block the interaction of CD47/SIRP $\alpha$ and TIGIT/PVR. The binding affinity of azelnidipine to SIRP $\alpha$ and PVR was tested, and the binding models were analyzed. The anti-tumor effects and potential mechanism of azelnidipine were also investigated both in vitro and in vivo. 


\section{Materials and Methods}

\subsection{Gene Expression Analysis}

The gene expression data of CD47 and PVR in the normal tissues and primary tumors of esophageal squamous cell carcinoma (ESCC) (GSE23400), colon tumor (GSE44076), and breast cancer (GSE42568) were downloaded from the public database National Institutes of Health Gene Expression Omnibus (GEO) (https:/ /www.ncbi.nlm.nih.gov/geo/, accessed on 3 May 2018, 6 March 2019, and 8 June 2020, respectively). The expression level of the target proteins was analyzed with the expression matrix and platform files.

\subsection{Virtual Screening}

The crystal structures of the human SIRP $\alpha$ (PDB ID: 2UV3) and TIGIT/PVR complex (PDB ID: 3UDW) were acquired from the Protein Data Bank database (PDB) (https: / www. rcsb.org/, accessed on 13 March 2018) [6,28]. The 3D structure of SIRP $\alpha$ (chain A of 2UV3) and PVR (chain C of 3UDW) was selected for docking followed by structure optimization with the software Molecular Operating Environment (MOE) (Chemical Computing Group ULC, Montreal, QC, Canada). Through the structure optimization, the target proteins were performed for protonation with the Protonate 3D function in MOE. The binding area was determined by searching the residues in the distance of $4.5 \AA$ and according to the references. The binding pocket for small molecule inhibitors was selected with the Site Finder module. A small molecule inhibitor library of FDA-approved drugs with 1686 compounds were performed the virtual screening (downloaded from the official website of Topscience (www.tsbiochem.com/), 10 May 2017). The compounds were prepared by removing extraneous salts or adjusting protonation states and minimization prior to molecular docking. Molecular docking with induced-fit parameter was conducted within $\mathrm{MOE}$. The candidate compounds were screened by a comprehensive analysis of the scores and interaction details with the pocket and especially the binding area. Twenty compounds were selected for the following screening tests.

\subsection{Cell Lines and Cell Culture}

Chinese hamster ovary cell line CHOK1 stably overexpressing human or murine CD47 and TIGIT (named as CHOK1-hCD47, CHOK1-mCD47, CHOK1-hTIGIT, CHOK1-mTIGIT, respectively) were established by lenti-viral transfection. CHOK1-derived cell lines, murine melanoma cell line B16-OVA fused with EGFP (GFP ${ }^{+}$B16-OVA), murine breast cancer cell line 4T1, and human colorectal cell line HT29; human esophageal squamous carcinoma cell line KYSE-70 and EC9706 were cultured in Roswell Park Memorial Institute (RPMI) 1640 medium. Human breast cancer cell line MCF-7, murine colorectal cancer cell lines CT26, MC38, and GFP ${ }^{+}$MC38 cells were cultured in Dulbecco's Modified Eagle Medium (DMEM). All the cells were cultured at $37{ }^{\circ} \mathrm{C}$ with $5 \% \mathrm{CO} 2$, under fully humidified conditions with medium supplemented with $10 \%$ fetal bovine serum (FBS) (BI, Kibbutz Beit Haemek, Israel) and antibiotics.

\subsection{Flow Cytometry Analysis}

Cells in good growth condition were harvested and used to detect the expression of CD47 and PVR. Antibodies anti-human CD47 APC, anti-human PVR PE, anti-mouseCD47 APC, anti-mouse-PVR APC, and matched isotype controls were purchased from eBioscience, San Diego, CA, USA. The effects of azelnidipine on the expression of CD47 and PVR was determined by incubating CT26 cells with $20 \mu \mathrm{M}$ of azelnidipine for $24 \mathrm{~h}$, or directly analyzing the tumor cells from tumor tissues of CT26-bearing mice treated with or without azelnidipine.

\subsection{Blocking Assays}

The abilities of small molecule inhibitors (SMIs) (purchased from TargetMol, Shanghai, China) to block the binding of SIRP $\alpha$ protein to CHOK1-CD47 cells or PVR protein to CHOK1-TIGIT cells were determined by flow cytometry, as previously reported [29]. The 
SMIs at indicated concentrations were incubated with Fc-tagged SIRP $\alpha$ or PVR protein for $30 \mathrm{~min}$ at $4^{\circ} \mathrm{C}$, followed by incubating with the indicated cells for another $30 \mathrm{~min}$. Human SIRP $\alpha$ protein (SIA-H5251, ACRO Biosystem, Beijing, China), mouse SIRP $\alpha$ protein (SIA-M5258, ACRO Biosystem, Beijing, China), human PVR protein (CD5-H82F6, ACRO Biosystem, Beijing, China), and mouse PVR protein (50259-M03H, Sino Biological, Beijing, China) were used. PE-conjugated anti-human IgG antibody was used to detect the FC protein bind to cells. Cells incubated with the Fc proteins and anti-human IgG antibody without the SMIs serve as the positive control. The mean fluorescent intensity (MFI) of each sample was detected and recorded by a flow cytometry. The blocking efficacy of the SMIs was calculated as the equation: (MFI of the positive control-MFI of the SMI)/MFI of the positive control $\times 100 \%$.

\subsection{Microscale Thermophoresis (MST)}

MST assays were performed to test the affinity of SMI-F11 (azelnidipine) to the indicated protein using the Monolith NT.115 system, as previously reported [30]. His tagged human SIRP $\alpha$ (SIA-H5225, ACRO Biosystem, Beijing, China), mouse SIRP $\alpha$ (50956M08H, Sino Biological, Beijing, China), human PVR (CD5-H5223, ACRO Biosystem, Beijing, China), and mouse PVR (50259-M08H, Sino Biological, Beijing, China) were used. The protein was labeled with Red-NHS647 dye (NanoTemper Technologies GmhH, Munich, Germany) according to the manufacturer's instructions. Azelnidipine was 2-fold serially diluted from $200 \mu \mathrm{M}$ and subsequently tested. Red-NHS647 dye-labeled protein was incubated with the diluted azelnidipine, with equal volume for $5 \mathrm{~min}$ at room temperature; the mixture was then loaded onto standard capillaries for detection. The KD values were calculated by the analysis software (MO. Affinity Analysis, version number 2.2.4, NanoTemper Technologies GmhH, Munich, Germany).

\subsection{Phagocytosis Assays}

Bone marrow-derived macrophage (BMDM) cells were isolated from C57BL/6 or BALB/c mice and induced with $20 \mathrm{ng} / \mathrm{mL}$ of GM-CSF (Peprotech, Rocky Hill, CT, USA) for 7 days. Meanwhile, the medium was replaced with fresh medium containing the cytokine and the adherent cells were harvested. Phagocytosis assays were performed by a co-culture of the BMDM macrophages with carboxyfluorescein succinimidyl ester-labeled $\left(\mathrm{CFSE}^{+}\right)$or $\mathrm{GFP}^{+}$tumor cells at a ratio of $1: 4$ in a serum-free medium at $37^{\circ} \mathrm{C}$ for $4 \mathrm{~h}$, in low-attachment 96-well tissue culture plates (Corning, New York, NY, USA). The cells were harvested and the macrophages were identified by a flow cytometry using anti-F4/80 antibody (eBioscience, San Diego, CA, USA). Then, 7-AAD (eBioscience) was used to exclude the dead cells. The effects of azelnidipine $(20 \mu \mathrm{M})$ on phagocytosis by BMDM cells were tested, and the anti-CD47 antibody (clone: miap301) were used as the positive control. Phagocytosis rate was determined with the formulation: $\mathrm{F} 4 / 80^{+} \mathrm{GFP}^{+}$or $\mathrm{F} 4 / 80^{+} \mathrm{CFSE}^{+}$ cells / Total F4/80+ cells.

\subsection{Tumor Models and Ex Vivo Assays}

BABL/c mice were subcutaneously (s.c.) injected with $2 \times 10^{5} \mathrm{CT} 26$ cells on the right back, and C57BL/ 6 mice were injected with $1 \times 10^{6}$ MC38 cells. One week later, tumorbearing mice were randomized into indicated groups, and intraperitoneal (i.p.) injected with azelnidipine or normal saline every day for two weeks. Tumor volumes were measured every other day by the (a) length, (b) width, and (c) height and calculated as volume $(\mathrm{V})=\mathrm{a} \times \mathrm{b} \times \mathrm{c} / 2$. For the combinational model of azelnidipine and radiotherapy (RT), MC38 tumor-bearing mice received irradiation (IR) when the tumors reached $80-100 \mathrm{~mm}^{3}$. The mice received one dose of $20 \mathrm{~Gy}$ irradiation at the tumor local site, and followed by the treatment of azelnidipine.

Tumor-bearing mice were sacrificed at the end of the treatment. The tumors were dissected and digested into single cell with an enzyme cocktail of $100 \mathrm{U} / \mathrm{mL}$ collagenase IV; and Dnase I. The infiltration of $\mathrm{CD}^{+} \mathrm{T}$ cells at the tumor site was detected by flow 
cytometry by staining of anti-mouse CD45, anti-mouse CD3, anti-mouse CD8 $\alpha$ or isotype control. The MDSCs at the tumor site were analyzed by staining of anti-mouse CD45, antimouse CD11b, anti-mouse Ly6C, and anti-mouse Ly6G. Spleens and draining lymph nodes were grinded and filtered into a single cell suspension. The tumor-infiltrating lymphocytes (TILs) isolated by Percoll-gradient centrifugation, splenocytes, and cells from draining lymph nodes were also performed the intracellular cytokine staining assays. The cells were stimulated with or without $20 \mathrm{ng} / \mathrm{mL}$ of phorbol 12-myristate 13-acetate (PMA, SigmaAldrich, St. Louis, MO, USA) and $1 \mu \mathrm{M}$ ionomycin (Sigma-Aldrich) with the presence of protein transport inhibitor cocktail (eBioscience) for $4 \mathrm{~h}$. The surface marker CD3, CD8, and the intracellular cytokine marker IFN- $\gamma$ were sequentially stained according to the Foxp3/Transcription Factor Staining Buffer Set (00-5523, eBioscience). The frequency of IFN- $\gamma$ secreting CD8 ${ }^{+} \mathrm{T}$ cells were counted according to the fluorescence minus one (FMO) control with the isotype control of IFN- $\gamma$ antibody.

To establish the CD8 ${ }^{+} \mathrm{T}$ cell depletion model, BALB/c mice were injected with $200 \mu \mathrm{g}$ of CD8-depleting antibody (clone: YTS169.4) or matched Rat IgG isotype control (SigmaAldrich) the day before tumor inoculation and as well as weekly thereafter, resulting in a total of 3 injections per mouse. Depletion efficacy of $\mathrm{CD}^{+} \mathrm{T}$ cells was verified by a flow cytometry with the blood sample of mice treated with indicated antibodies for 7 days.

\subsection{Statistical Analysis}

Statistical analysis was performed with paired or unpaired two-tailed Student's $t$-test or two-way ANOVA with Tukey's multiple comparisons test for analyzing differences between groups of quantitative data represented as means \pm SEM, with significant differences marked on the figures. Significance levels were defined as ${ }^{*} p<0.05,{ }^{* *} p<0.01$, *** $p<0.001$.

\section{Results}

\subsection{CD47 and PVR Are Over-Expressed in Tumor Tissues and Cell Lines}

CD47 has been reported as an important therapeutic target, and its ligation with the ligand $\operatorname{SIRP} \alpha$ plays critical roles in the innate immunity [31]. The immune checkpoint TIGIT, an acknowledged exhaustion maker of both NK cell and effector CD8 ${ }^{+} \mathrm{T}$ cells, exerts inhibitory signals by interacting with its major ligand PVR [3]. The CD47/SIRP $\alpha$ and TIGIT/PVR signaling pathway jointly contribute to the immunosuppressive tumor microenvironment [32]. An increasing number of researches reported that CD47 and PVR over-expressed in various tumors. Consistent with previous reports in the public database The Cancer Genome Atlas (TCGA), CD47 and PVR expressed at high levels in Esophageal Cancer (ESCA), Colon Cancer (COAD), Head and Neck Cancer (HNSC), and Stomach Cancer (STAD) analyzed by the online tool TIMER [12,33-35]. Through the analysis of the public GEO database, the evaluated expression level of CD47 and PVR was also validated in esophageal squamous cell carcinoma, colon cancer, and breast cancer tissues in the GSE23400, GSE44076, and GSE42568, respectively (Figure 1A-C).

We also investigated the expression of CD47 and PVR on the tumor cell lines by flow cytometry. CD47 and PVR were highly co-expressed on the human esophageal squamous cell lines KYSE-70 and EC9706, the colorectal cancer cell line HT29, and the breast cancer cell line MCF7 (Figure 1D). The targets were also highly co-expressed in murine colorectal cancer cell lines MC38 and CT26, the metastatic breast cancer cell line 4T1, and the malignant melanoma cancer cell line B16-OVA (Figure 1E). Collectively, consistent with the previous literature, our results revealed the over-expression of CD47 and PVR in tumors, suggesting the promising role of CD47 and PVR in cancer immunotherapy. 

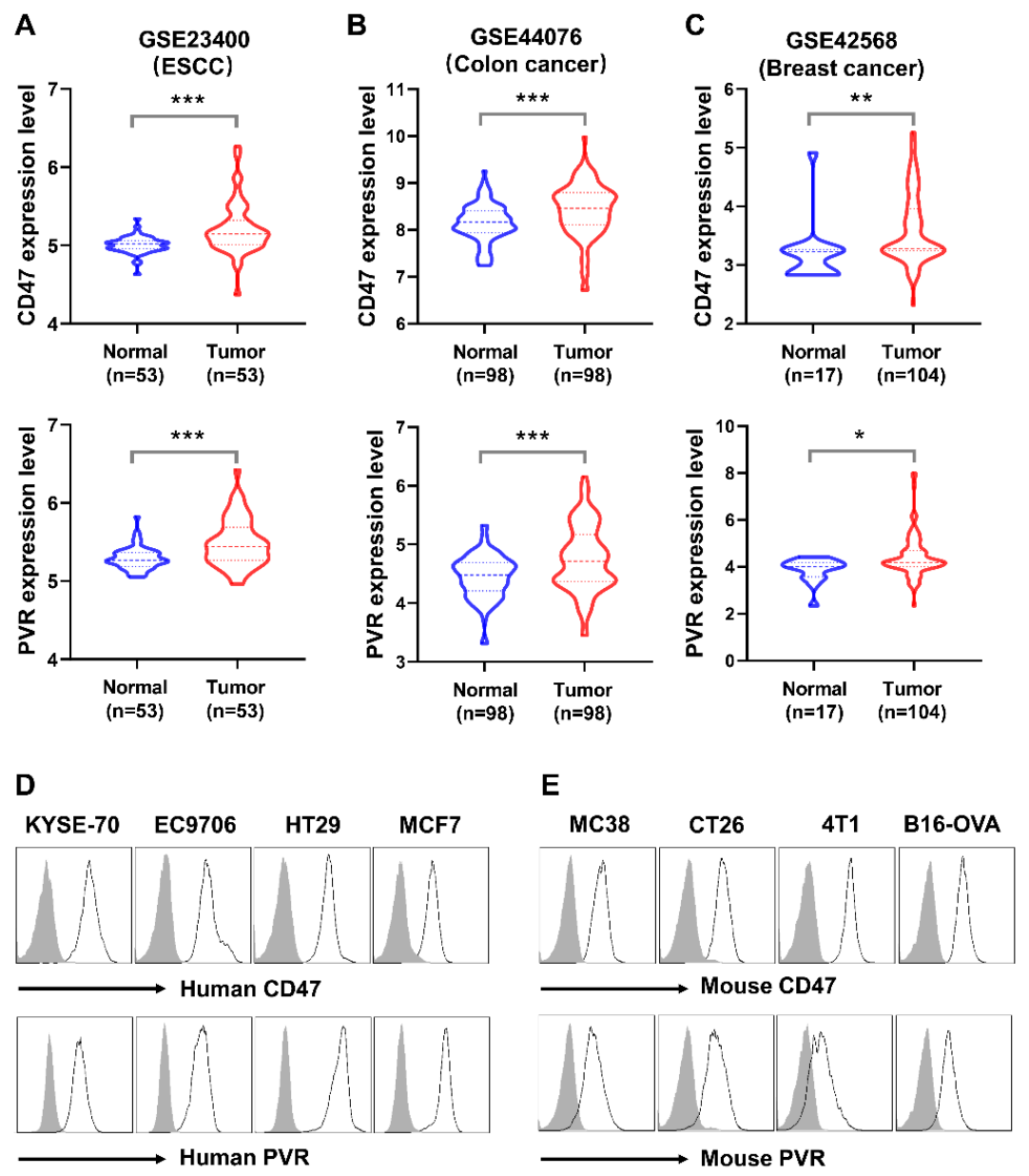

Figure 1. The expression of CD47 and PVR in tumor tissues and cell lines. (A) The expression level of CD47 and PVR in GSE23400 with the esophageal squamous cell carcinoma (ESCC) and paired normal tissues are represented. (B) The expression level of CD47 and PVR in GSE44076 with the colon cancer and paired normal tissues are represented. (C) The expression level of CD47 and PVR in GSE42568 with the breast cancer and normal tissues are represented. The sample numbers are shown in the indicated groups. The statistical analysis was conducted with paired Student's $t$-test for the cohorts GSE23400 and GSE44076, and unpaired Student's $t$-test for the cohort GSE42568. ${ }^{*} p<0.05,{ }^{* *} p<0.01,{ }^{* * *} p<0.001$. (D) Flow cytometry analysis of CD47 and PVR on human tumor cell lines. (E) Flow cytometry analysis of CD47 and PVR on murine tumor cell lines. The histogram lines represent indicated antibodies; shaded histogram represents the matched isotype controls.

\subsection{Discovery of Small Molecule Inhibitors Targeting CD47/SIRP $\alpha$ and TIGIT/PVR Pathways by} Virtual Screening

The clinical trial of the dual-target small molecules CA-170 (targeting PD-L1 and VISTA) and CA-327 (targeting PD-L1 and TIM-3) suggested the successful development of dual-target small molecule inhibitors targeting different immune checkpoints [36,37]. Dual-target small molecule inhibitor (SMI) co-targeting the CD47/SIRP $\alpha$ and TIGIT/PVR may also exert exciting effects in the cancer immunotherapy. The high-throughput virtual screening of small molecules with the molecular docking could facilitate the drug discovery. The high-resolution structures of CD47/SIRP $\alpha$ in monomer or complexes have been resolved by many groups, providing the structural basis for the virtual screening [28,38]. Considering that targeting CD47 may have relative high toxicity in the blood [39], $\operatorname{SIRP} \alpha$ was selected as the target for screening. CD47 binds to SIRP $\alpha$ at a surface constituted by the $B C, C^{\prime} D, D E$, and FG loops represented as magenta (Figure 2A), unlike most surface proteins that use the folding surfaces to interact with each other. The four loops form a large pocket suitable for the binding of small molecules, as the largest pocket determined 
by MOE (presents as cyan) overlays most of the CD47/SIRP $\alpha$ binding area, which was selected for the following virtual screening. As with most of the immunoglobulin superfamily surface proteins, TIGIT and PVR mutually interact through the surface formed by the $\beta$-sheets. The TIGIT binding area on PVR formed by the C, $C^{\prime}, C^{\prime \prime}, F$ and $G$ sheet was labeled as magenta (Figure 2B). Although the binding area is relatively smooth and not optimal for small molecule occupancy, a binding pocket close to, and partially overlapping with, the key residues on the $C, C^{\prime}$ sheet and $C^{\prime}$ loop has proved to be feasible for the inhibition of protein-protein interaction (Figure 2B) [12]. The structure of the target proteins SIRP $\alpha$ and PVR were further processed, and the indicated pockets were set to perform the molecular docking. The protein and the small molecules from the FDA-approved drug library were prepared and the small molecules were screened according to the procedures illustrated in Figure 2C. Finally, 20 candidate small molecules were obtained with the comprehensive consideration of the docking parameters (Supplemental Table S1).

A

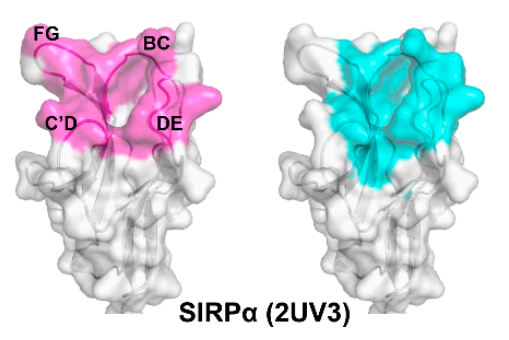

B

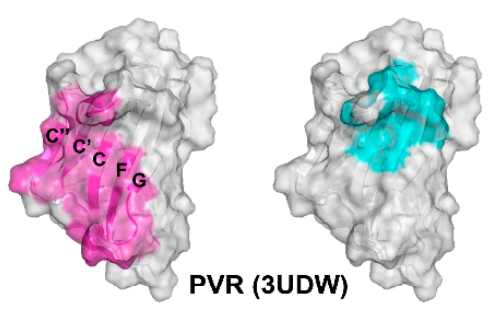

C

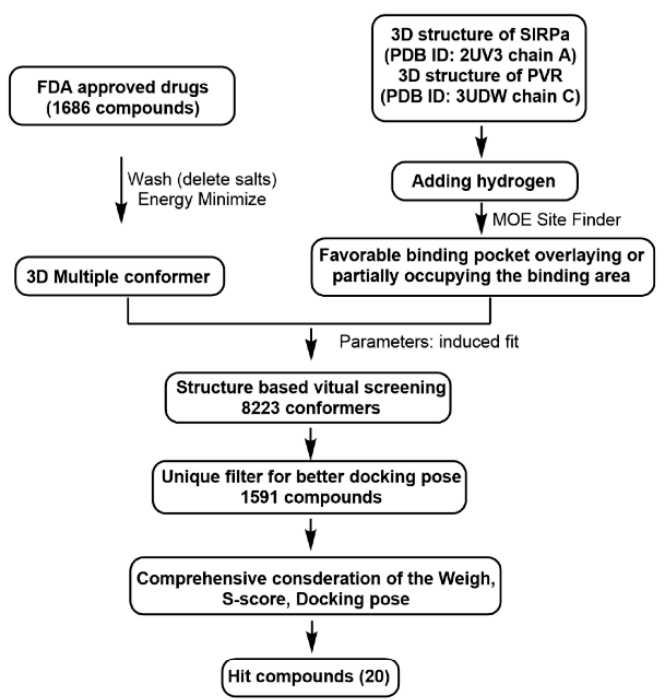

F

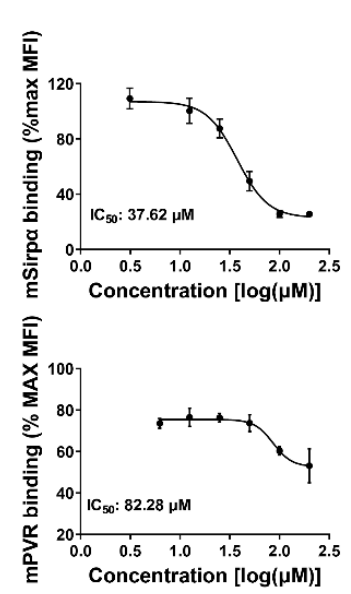

Figure 2. Virtual screening of small molecule inhibitors targeting SIRP $\alpha$ and PVR from the FDA-approved drugs. (A) The structure of SIRP $\alpha$ from PDB (PDB ID: 2UV3 chain A). The magenta represents the binding area of CD47 on SIRP $\alpha$, the cyan represents the pocket for small molecule inhibitors calculated by MOE. (B) The structure of PVR from PDB (PDB ID: 3UDW chain C). The magenta represents the binding area of TIGIT on PVR, the cyan represents the pocket for small molecule inhibitors calculated by MOE. (C) The virtual screening procedures of the inhibitors. (D) The blocking efficacy of the small molecule inhibitors of a preliminary test at a single concentration of $100 \mu \mathrm{M}$. (E) The blocking efficacy of azelnidipine targeting human CD47/SIRP $\alpha$ and TIGIT/PVR. (F) The blocking efficacy of azelnidipine targeting mouse CD47/SIRP $\alpha$ and TIGIT/PVR. The data are presented as mean \pm SEM and are representative of at least three independently performed experiments. 


\subsection{Screening and Validation of the Candidate Small Molecule Inhibitors by Blocking Assay}

The small molecule inhibitor could not only bind to the target; most importantly, it should interfere with the receptor-ligand interactions. Subsequently, the candidate inhibitors were screened by the blocking assays with the established CD47 and TIGIT over-expressing cell lines. The test was initialed with a preliminary screening of the candidates with a single concentration (Figure 2D). Generally, the SMI blocking efficacy to the CD47/SIRP $\alpha$ interaction was much higher than that of the TIGIT/PVR. Small molecule inhibitors SMI-F11, SMI-F12, SMI-F17, and SMI-F19 could block the interaction of CD47 and SIRP $\alpha$ with more than 50\% blocking efficacy, while SMI-F11, SMI-F16, SMIF17, and SMI-F20 show better activities in interfering with the TIGIT/PVR interaction. Therefore, the SMI-F11 and SMI-F17 may exert dual blocking effects in the CD47/SIRP $\alpha$ and TIGIT/PVR pathways. Blocking assays with a concentration gradient of the SMI were further performed to validate the preliminary screening. SMI-11 (azelnidipine) could efficiently block both the human CD47/SIRP $\alpha$ and TIGIT/PVR interactions with the IC 50 of about $36 \mu \mathrm{M}$ and $28 \mu \mathrm{M}$, respectively (Figure 2E), while SMI-17 could not exert blocking effects in a concentration-dependent manner. The similar blocking assays with the mouse CD47 and TIGIT over-expressing cells were also performed. Azelnidipine could also block both the mouse CD47/SIRP $\alpha$ and TIGIT/PVR interactions with the $\mathrm{IC}_{50}$ of about $37 \mu \mathrm{M}$ and $82 \mu \mathrm{M}$, respectively (Figure $2 \mathrm{~F}$ ). Considering that most pairs of the immune checkpoints interact with the corresponding ligand in parallel forms, the blocking assays with the PD1/PD-L1 interaction system was also conducted to test the specificity. Azelnidipine could not block the interaction of human PD-1/PD-L1, suggesting that azelnidipine has specificity by blocking the CD47/SIRP $\alpha$ and TIGIT/PVR interactions (Supplemental Figure S1).

\subsection{The Binding Activity and Model of Azelnidipine to SIRP $\alpha$ and PVR}

After determining that azelnidipine could dually block both the CD47/SIRP $\alpha$ and TIGIT/PVR pathways, its affinity to the targets SIRP $\alpha$ and PVR was further determined using the MST method. Azelnidipine could bind to human SIRP $\alpha$ and PVR with the comparable $\mathrm{K}_{\mathrm{D}}$ values of $5.37 \mu \mathrm{M}$ and $6.48 \mu \mathrm{M}$ (Figure 3A,B). Considering the high sequence identity and similar structure of the human and mouse SIRP $\alpha$ or PVR, MST assays was also conducted to test the binding of azelnidipine to the mouse proteins. Azelnidipine could bind the mouse SIRP $\alpha$ with a $K_{D}$ value of $3.75 \mu \mathrm{M}$ comparable to human SIRP $\alpha$, while it binds to mouse PVR with a $K_{D}$ value of $13.28 \mu \mathrm{M}$ (Figure 3C,D). The binding of azelnidipine to mouse PVR is slightly weaker, and this may explain the difference of the blocking efficacy between the human and mouse TIGIT/PVR.

To elucidate the possible mechanism of the dual targeting of azelnidipine to SIRP $\alpha$ and PVR, the binding models were analyzed. The convoluted interacting face of CD47 with several $\beta$-sheets and loops occupy the surface of SIRP $\alpha$ comprised of the BC, $C^{\prime} D, D E$, and FG loops. With analysis of the docking results, azelnidipine interacts with the residues G34 on the BC loop and T67 on the DE loop of SIRP $\alpha$, among which T67 was critical for receptor-ligand ligation by interacting with CD47 through the residues N27 on the BC loop and R103 on the G $\beta$-sheet (Figure 3E). By interacting with SIRP $\alpha$ across BC and DE loops, azelnidipine could partially occupy the binding area on $\operatorname{SIRP} \alpha$ and interfere the CD47/SIRP $\alpha$ ligation. Azelnidipine could also nicely target the TIGIT binding area on PVR and form a barrier for the protein-protein interaction. In detail, azelnidipine interacts with the residues G73 on the CC' loop and S132 on the G $\beta$-sheet (Figure 3F). Although TIGIT binds to PVR without interacting with G73, the S72 and S74 are involved in the formation of the key residue pairs, and the interaction between azelnidipine and G73 forms steric hindrance for TIGIT/PVR ligation. The residue S132 on PVR interacts with the N58 on TIGIT, and functions as a critical residue for TIGIT/PVR interaction [40]. 

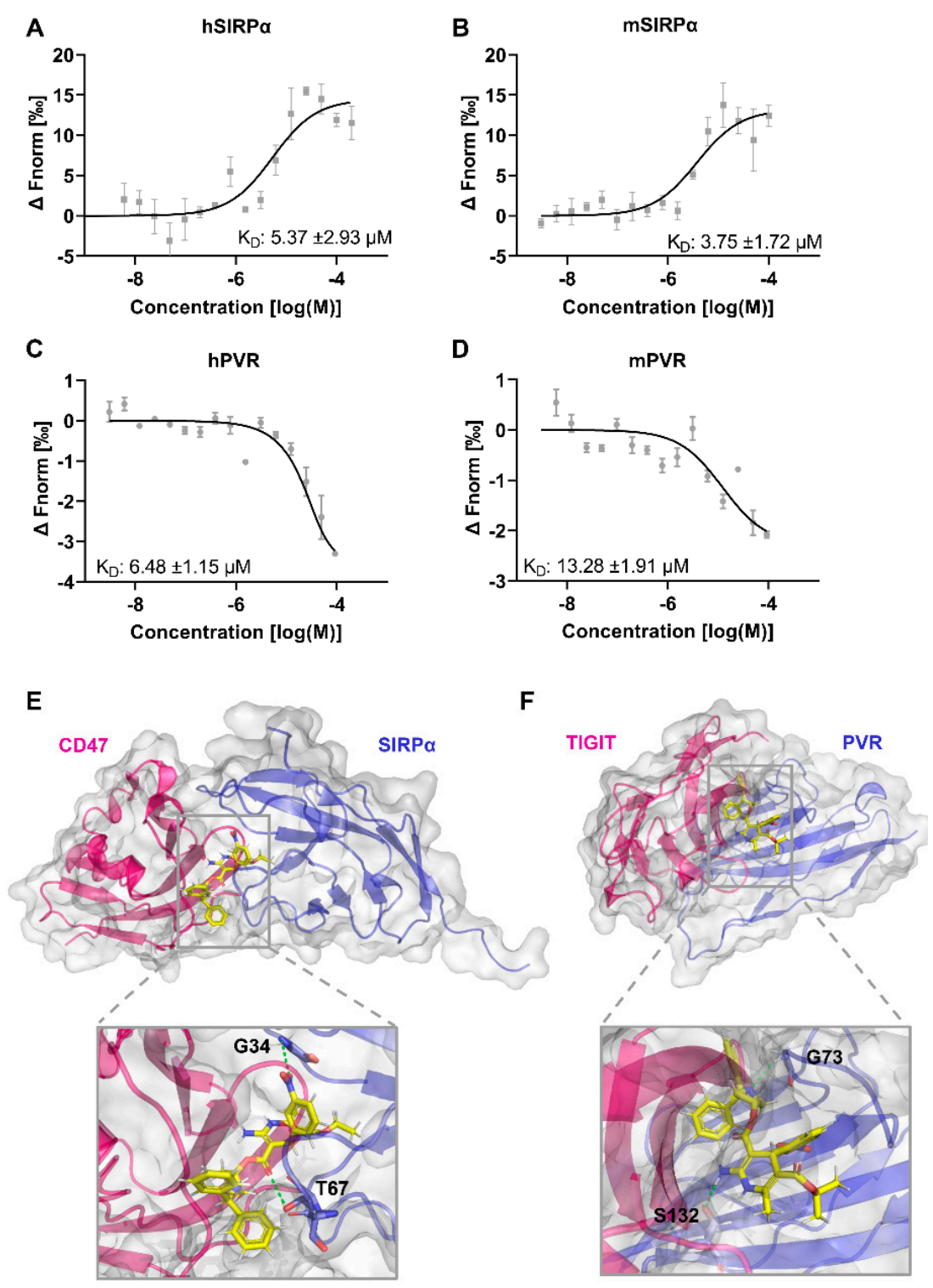

Figure 3. The binding affinity and model of azelnidipine to SIRP $\alpha$ and PVR. Binding of azelnidipine to human and mouse SIRP $\alpha$ or PVR was examined by the MST. $(\mathbf{A}, \mathbf{B})$ Dose response curves of azelnidipine binding to human $\operatorname{SIRP} \alpha(\mathbf{A})$ and PVR (B). (C,D) Dose response curves of azelnidipine binding to mouse SIRP $\alpha(C)$ and PVR (D). The $K_{D}$ values were calculated with analysis software (MO. Affinity Analysis v2.2.4). Data are representative of at least three independent experiments. (E) The binding model and detail of azelnidipine to SIRP $\alpha$. The protein surface of CD47 (magenta) and SIRP $\alpha$ (blue) and azelnidipine (yellow) are shown, with the interactions labeled. (F) The binding model and detail of azelnidipine to PVR. The protein surfaces of TIGIT (magenta) and PVR (blue) and azelnidipine (yellow) are shown, with the interactions labeled.

\subsection{Azelnidipine Could Enhance the Macrophages-Mediated Phagocytosis of Tumor Cells In Vitro}

CD47 mediates tumor evasion by serving as a "do not eat me" marker and transmitting the anti-phagocytic signal to macrophages. Macrophages-mediated phagocytosis was one of the major mechanisms of CD47 targeted therapies. Here, the effects of azelnidipine on the macrophage phagocytosis of tumor cells were explored by a co-culture of the BMDM cells and various types of tumor cells. As a result, a blockade of CD47/SIRP $\alpha$ interaction by azelnidipine could significantly enhance the phagocytosis of the MC38, CT26, and B16-OVA cells by BMDM cells slightly inferior to the anti-CD47 positive control (Figure 4). 
A

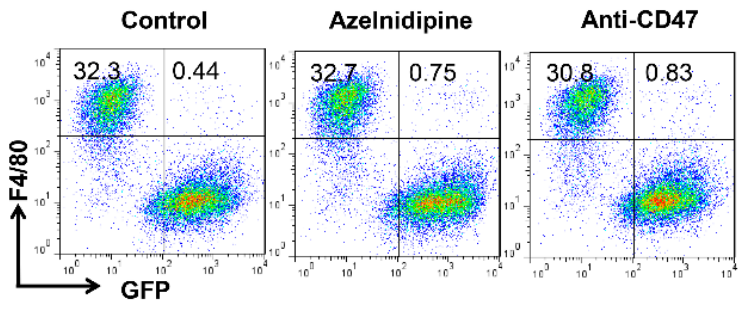

B

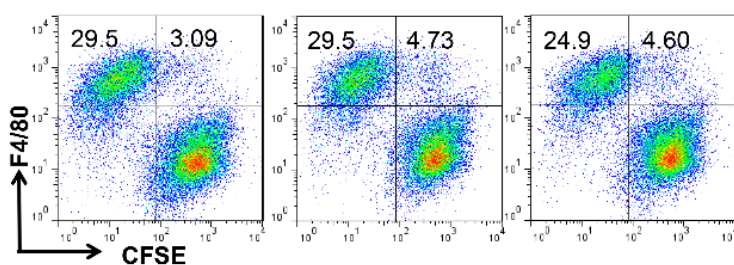

C

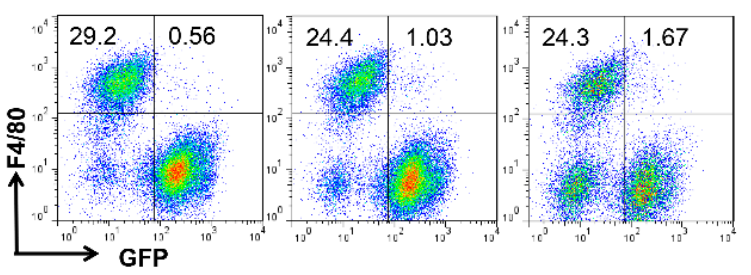

MC38

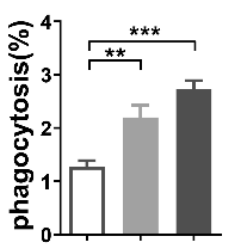

CT26

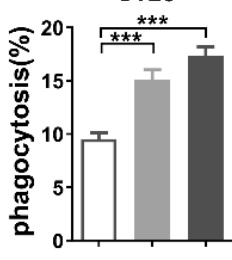

B16-OVA

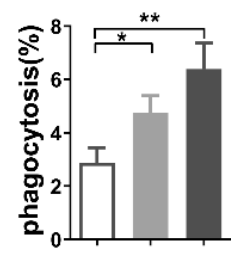

Figure 4. Azelnidipine enhances macrophage mediated phagocytosis of the tumor cells. The effects of the azelnidipine on the phagocytosis of the macrophages were tested by a co-culture of the tumor cells and BMDM cell derived from C57BL/ 6 or BALB/c mice. GFP ${ }^{+}$MC38 cells (A), CFSE-labeled CT26 cells (B), and GFP ${ }^{+}$B16-OVA (C) were co-cultured with the BMDM cells at the ratio of 4:1 in serum-free medium at $37^{\circ} \mathrm{C}$ for $4 \mathrm{~h}$ with or without the presence of $20 \mu \mathrm{M}$ of azelnidipine or the positive control anti-CD47 antibody. $\mathrm{GFP}^{+}$or $\mathrm{CFSE}^{+} \mathrm{BMDMs}$ were detected by a flow cytometry. Representative flow cytometry plots and the summary data of at least three independent experiments were shown. ${ }^{*}, p<0.05,{ }^{* *}, p<0.01,{ }^{* * *}, p<0.001$, Student's $t$-test.

\subsection{Azelnidipine Could Inhibit MC38 Tumor Growth Combined with Radiotherapy}

The effects of a CD47/SIRP $\alpha$ blockade by azelnidipine were further explored in the MC38 tumor model, which has a large quantity of intratumoral macrophages and was widely used for the CD47 targeted anti-tumor study [18]. Tumor-bearing mice with visible tumors of about $100 \mathrm{~mm}^{3}$ were intraperitoneally treated with 2 and $5 \mathrm{mg} / \mathrm{kg}$ of azelnidipine. Both dosages of azelnidipine could significantly restrict the growth of MC38 tumors (Figure 5A). At the clinic, radiotherapy is frequently adopted for the tumor eradication. Moreover, the tumor local irradiation (IR) could augment the infiltration of monocytic myeloid-derived suppressor cells (M-MDSCs) $\left(\mathrm{CD}^{2} 1 \mathrm{~b}^{+} \mathrm{Ly}_{6 \mathrm{C}}{ }^{\mathrm{hi}} \mathrm{Ly}_{6 \mathrm{G}}{ }^{-}\right)$in tumor tissues [41]. The M-MDSCs could function themselves or differentiated into macrophages to phagocytize tumor cells $[42,43]$. We therefore hypothesized that pretreatment of local IR might synergize with azelnidipine to further enhance tumor control. Tumor-bearing mice received locally IR at 20 Gy when the tumors reached approximate $100 \mathrm{~mm}^{3}$, and followed by daily treatment of azelnidipine. Consistent with our inference, a combination of azelnidipine and IR could remarkably suppress tumor growth (Figure 5B). Mechanistically, the analysis of the tumor infiltrating immune cells confirmed that local IR of MC38 tumors indeed significantly augment the frequency of Ly6G-Ly6C ${ }^{+} \mathrm{M}-\mathrm{MDSC}$, with or without the combination of azelnidipine (Figure 5C). The expression level of SIRP $\alpha$ on the intratumoral M-MDSCs of tumor-bearing mice received local IR also significantly increased (Figure 5D). Irradiation damages the tumor tissues and increases infiltration and SIRP $\alpha$ expression on MDSCs, and combined with azelnidipine that blocks CD47/SIRP $\alpha$ interaction, it can enhance MDSC cell-mediated phagocytosis of tumor cells, thereby remarkably inhibiting the tumor growth. 
A

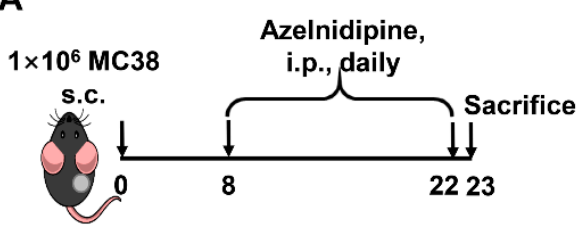

B

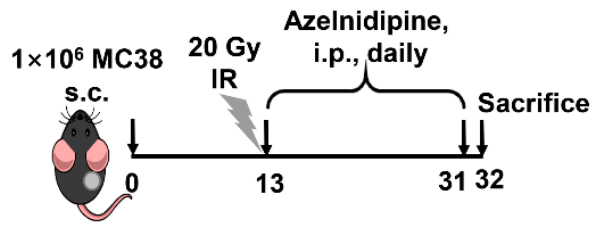

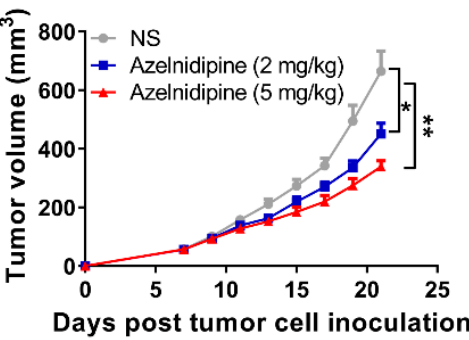

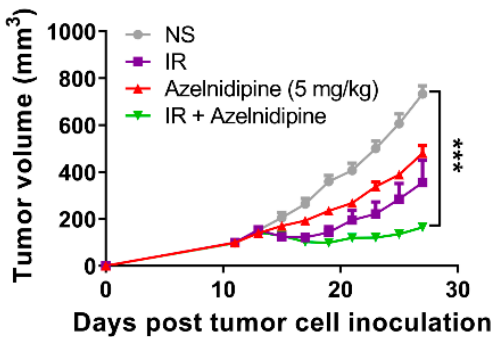

C

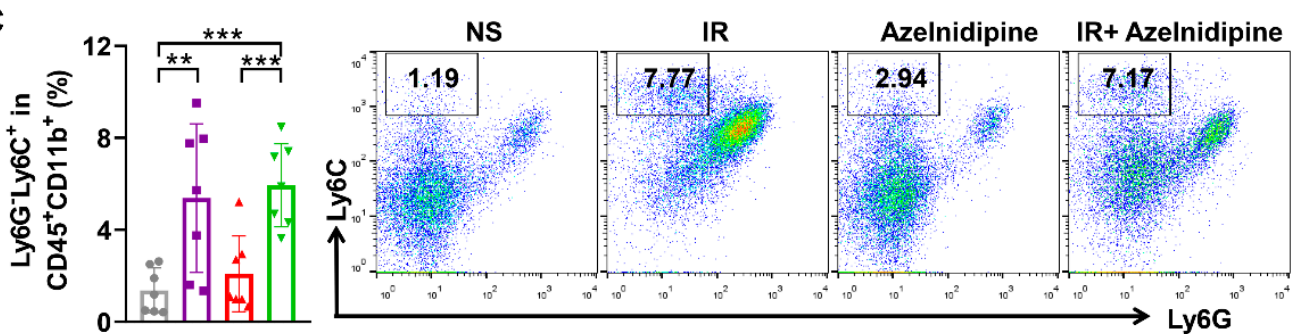

D

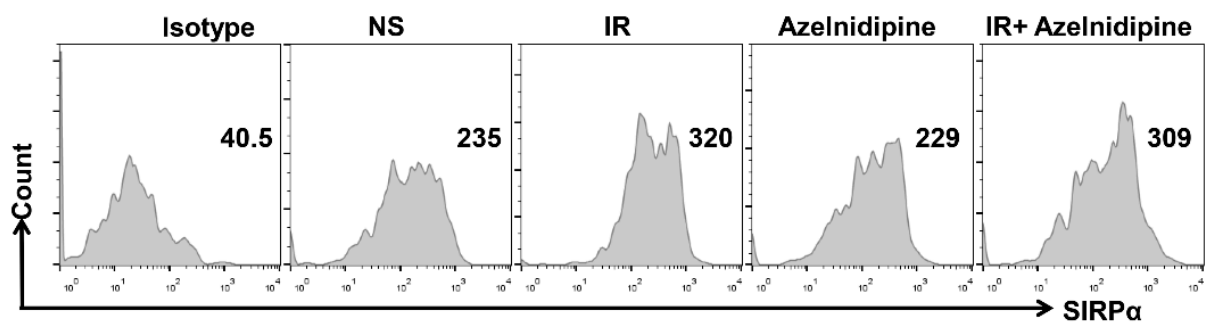

Figure 5. The antitumor effects of azelnidipine alone or combined with radiotherapy in MC38 tumor model. Azelnidipine alone or combined with radiotherapy significantly inhibit MC38 tumor growth. (A) Mice were i.p. injected with 2 or $5 \mathrm{mg} / \mathrm{kg}$ of azelnidipine each day for 2 weeks as in the schematic diagram. Tumor growth curve of MC38 tumor-bearing mice treated with normal saline or azelnidipine alone $(n=9$ or 10). (B) Mice were i.p. injected with $5 \mathrm{mg} / \mathrm{kg}$ of azelnidipine each day followed by $20 \mathrm{~Gy}$ of local IR as in the schematic diagram. Tumor growth curve of MC38 tumor-bearing mice treated with normal saline, IR, azelnidipine alone or combined $(n=7)$. (C) Representative flow cytometry plots and summary data of the MDSCs in the tumor tissues of each group $(n=7)$. (D) Representative flow cytometry histograms of the expression of SIRP $\alpha$ on MDSCs in the tumor tissues of each group $(n=7) .{ }^{*}, p<0.05,{ }^{* *}, p<0.01,{ }^{* * *}, p<0.001$. Two-way ANOVA with Tukey's multiple-comparisons test $(\mathbf{A}, \mathbf{B})$ and Student's $t$-test $(\mathbf{c})$ were conducted.

\subsection{Azelnidipine Could Significantly Inhibit the Tumor Growth and Elicit Anti-Tumor T Cell Immune Response}

It is reported that a CD47/SIRP $\alpha$ blockade and TIGIT/PVR blockade could elicit significant anti-tumor responses $[11,44]$. We further investigated the anti-tumor effects of azelnidipine in a CT26 model, which is widely used for CD47/SIRP $\alpha$ and TIGIT/PVR blockade therapy and the exploration of the $\mathrm{T}$ cell immune response. By targeting PVR, azelnidipine could significantly restrict the CT26 tumor growth at both administration dosages (Figure 6A,B). Azelnidipine treatment could also remarkably increase the frequency of $\mathrm{CD}^{+} \mathrm{T}$ cells in the tumor tissues (Figure $6 \mathrm{C}$ ). More importantly, except the increase in cell number, the IFN- $\gamma$ secretion function of the tumor infiltrating $\mathrm{CD} 8^{+} \mathrm{T}$ cells also enhanced (Figure 6D). CD8 ${ }^{+} \mathrm{T}$ cells are the main effector anti-tumor cells; thus, the augment quantity 
and enhanced function of the $\mathrm{CD} 8^{+} \mathrm{T}$ cells might jointly lead to the tumor reduction. The systemic immune response was also explored; a low dosage of azelnidipine could enhance the secretion of $\mathrm{CD}^{+} \mathrm{T}$ cells in the spleen, while a high dosage of azelnidipine could significantly boost the secretion of IFN- $\gamma$ in both the spleen and the tumor draining lymph node (Figure 6E,F). Collectively, the above results proved that targeting PVR with azelnidipine could significantly inhibit the tumor growth and elicit anti-tumor $\mathrm{T}$ cell immune response.

A

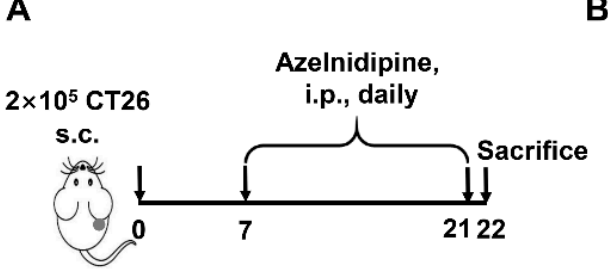

C

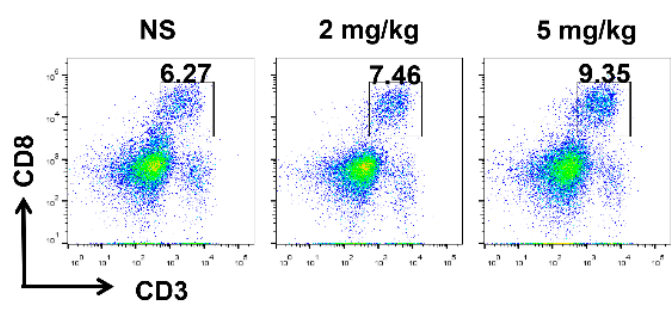

D

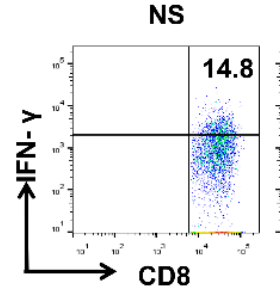

E

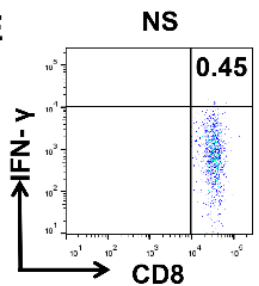

NS

F

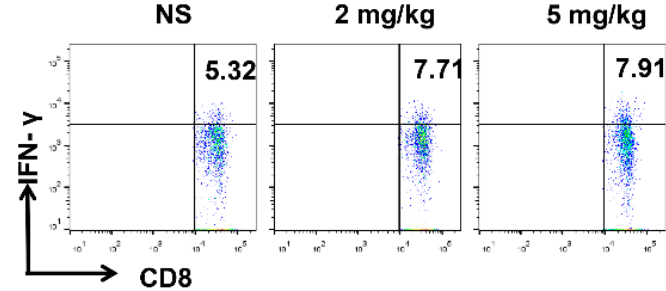

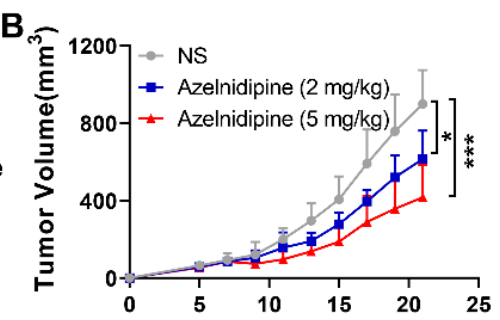

Days post tumor cell inoculation
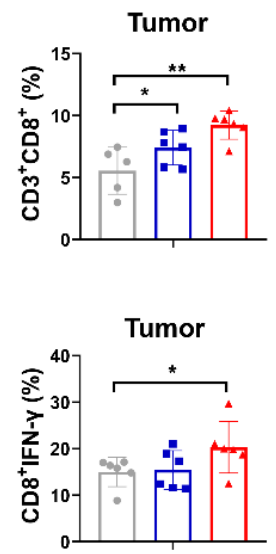

dLN
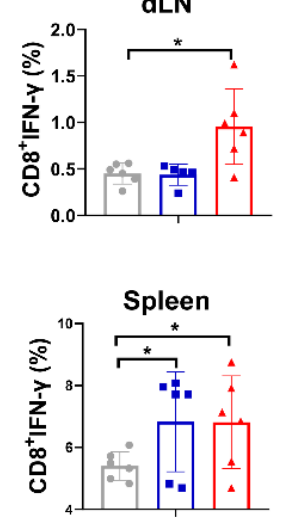

Figure 6. The antitumor effects of azelnidipine in CT26 tumor model. (A) CT26 tumor-bearing mice were i.p. administrated with 2 or $5 \mathrm{mg} / \mathrm{kg}$ of azelnidipine each day for 2 weeks as in the schematic diagram. (B) Tumor growth curve of CT26 tumor-bearing mice treated with normal saline or azelnidipine $(n=7) . *, p<0.05,{ }^{* * *}, p<0.001$.Two-way ANOVA with Tukey's multiple-comparisons test were conducted. (C) Representative flow cytometry plots and summary data of the frequency of $\mathrm{CD}^{+} \mathrm{T}$ cells in the tumor tissues. Representative intracellular IFN- $\gamma$ staining plots and summary data of the tumor infiltrating $\mathrm{CD} 8^{+} \mathrm{T}$ cells $(\mathbf{D})$, tumor-draining lymph node cells $(\mathbf{E})$ and splenocytes (F). The frequency of IFN- $\gamma$ secreting $\mathrm{CD}^{+} \mathrm{T}$ cells were counted according to the fluorescence minus one (FMO) control with the isotype control of IFN- $\gamma$ antibody. ${ }^{*}, p<0.05,{ }^{* *}, p<0.01$, Student's $t$-test were conducted. 
Since the function of $\mathrm{CD} 8^{+} \mathrm{T}$ cells significantly enhanced with the azelnidipine treatment, we further explored whether the anti-tumor effects of azelnidipine depends on the $\mathrm{CD}^{+} \mathrm{T}$ cells. A CD8 ${ }^{+} \mathrm{T}$ cells depletion CT26 tumor model was well established (supplemental Figure S2a,b). In the Rat IgG treated groups, azelnidipine could significantly inhibit tumor growth as in Figure 6B, while the anti-tumor effect was abrogated with $\mathrm{CD} 8^{+} \mathrm{T}$ cells depleted (supplemental Figure S2c). These results suggested that the anti-tumor effects of azelnidipine was $\mathrm{CD}^{+} \mathrm{T}$ cell dependent, as with most immune checkpoint blockers.

Furthermore, we also analyzed the infiltration of MDSCs and the expression of SIRP $\alpha$ with the CT26 tumor-bearing mice, same as in the MC38 tumor model in Figure 5. Consistent with Figure 5, there are large quantity of MDSCs with a high expression level of SIRP $\alpha$ in the tumor tissues (supplemental Figure S2d,e). Although the infiltration of MDSCs and their expression level of SIRP $\alpha$ show no significant difference in the NS and azelnidipine groups, the inhibitory pathway of CD47/SIRP $\alpha$ exists in the CT26 tumor model, which provides the rationality for a CD47/SIRP $\alpha$ blockade. We also explored the effects of azelnidipine on the expression of CD47 and PVR. In vitro, azelnidipine could slightly inhibit the expression of PVR on CT26 cells without suppressing the expression of CD47 analyzed by a flow cytometry (supplemental Figure S3a,b). Meanwhile, we also analyzed the expression of CD47 and PVR on CT26 tumor cells derived from the tumor tissues. Consistent with the results in vitro, azelnidipine could inhibit the expression of PVR but not that of CD47 (Supplemental Figure S3c,d). Thus, azelnidipine could not only block the TIGIT/PVR pathway, but also reduce the expression of PVR.

\section{Discussion}

$\mathrm{CD}^{+} \mathrm{T}$ cells are important effector cells in anti-tumor immunity, while innate immunity is indispensable to initiate adaptive immunity and recruit activated $T$ cells to reach the action site. The success of bispecific antibody targeting of both innate and adaptive immune checkpoints has firmly established the proof of the concept that combinational therapy of the innate and adaptive immunity has a broad application prospect [17]. A CD47/SIRP $\alpha$ blockade not only re-motivates the innate immunity by enhancing the macrophages phagocytosis, but also enhances the adaptive immunity by cross-priming T cells by DC cells [44]. A TIGIT/PVR blockade could reverse the exhaustion of both T cells and NK cells, resulting in significant and durable anti-tumor effects [3]. Combinational therapy targeting $\mathrm{CD} 47 / \mathrm{SIRP} \alpha$ and TIGIT/PVR pathways is of important clinical significance.

Although CD47 was upregulated on tumor cells, its ubiquitous expression on the normal cell, especially the hematopoietic cells, has been a major concern for CD47-targeted therapy. The side effects of hematological toxicity caused by non-specific clearance with the anti-phagocytic signal released may severely restrict the application of a CD47 blockade. With relatively restricted expression, an SIRP $\alpha$ blockade could enhance the macrophage mediated innate immunity with reduced side effects such as anemia. Therapeutics targeting SIRP $\alpha$ including antibodies and macrocyclic peptide are rapidly developing [45]. PVR, the major ligand of TIGIT, plays a pivotal role in multitudinous biological processes, especially the tumor immune escape. From the resolved structure, PVR shows advantages for drug screening with a relative rough surface compared with TIGIT [12]. On the other hand, as a shared ligand, a PVR blockade is expected to interfere in both TIGIT/PVR and CD96/PVR negative signal pathways [46]. Collectively, SIRP $\alpha$ and PVR was selected as the targets.

Drug repositioning plays an increasingly important role in the field of cancer immunotherapy. Numerous methods or tools are developed to accelerate the identification of new drug-target interactions or new target-disease relations, among which computational molecular docking based on the structures to predict binding site complementarity between the ligand and target is a promising approach [22]. Resolution of the crystal structure of the immune checkpoints makes it feasible to acquire small molecule inhibitors by docking-based virtual screening [6,38]. In this study, compounds approved by the FDA were screened by docking to the potential binding pockets of SIRP $\alpha$ and PVR identified with the structural analysis of SIRP $\alpha$ and PVR. Excitingly, the FDA-approved drug azelni- 
dipine could occupy the binding areas of CD47/SIRP $\alpha$ and TIGIT/PVR and interact with the key residues.

The mainstream strategy for an immune checkpoint blockade is the specific antibodies. Low molecular weight inhibitors, including high-affinity protein mutants, peptides, and small molecules, have also been designed for cancer treatment [37]. Large numbers of small molecule inhibitors targeting PD-1/PD-L1 have been developed. Using virtual screening in combination with comprehensive methods, we redefined the function of the small molecule compound liothyronine in blocking the interaction between TIGIT and PVR for cancer immunotherapy [12]. Furthermore, a single drug with function in modulating the activity of multiple targets over single-targeted or combination therapy has potential advantages. Multiple target molecules with dual activity shows a more predictable pharmacokinetic profile and less toxicity than a cocktail of multiple molecules [47]. Small molecule CA-170 for a dual blockade of PD-L1 and VISTA, and CA-327 for a dual blockade of PD-L1 and TIM3 have been proceeded to clinical trials [36]. Here, we firstly demonstrated that azelnidipine dually targeting SIRP $\alpha$ and PVR could be a promising anti-tumor modality in cancer immunotherapy. Although the effect of azelnidipine for dual blocking of CD47/SIRP $\alpha$ and TIGIT/PVR interactions indeed occurs within one single model, it is hard to distinguish the contribution to the anti-tumor effects of either single blockade. In addition, since there are currently no specific small molecule inhibitors for TIGIT/PVR and CD47/SIRP $\alpha$, it is not possible to compare the effect of a single blockade of azelnidipine for the time being.

Calcium channel blockers are widely used for the treatment of hypertension as a first-line drug and have been reported to have immunosuppressive roles in inhibiting the functions of T cells and macrophages [48-50]. Other researches demonstrated that different calcium channel blockers have different regulatory effects on the cytokine secretion of peripheral blood mononuclear cells [51]. The research about the immune-regulatory role of azelnidipine is limited and controversial. On the one hand, azelnidipine was reported to inhibit the differentiation and activation of THP- 1 macrophages and the cytokine secretion of peripheral blood mononuclear cells $[52,53]$. It has also been reported that azelnidipine can enhance the secretion of IL-12 p40 mediated by immune complex, without facilitating the differentiation of Th17 cells, which induces autoimmunity or impair anti-tumor immunity [54]. Despite the dispute and requirement of further exploration, azelnidipine has been reported to possess anti-tumor effects $[55,56]$. Azelnidipine can inhibit the growth of various tumor cells in vitro, and significantly inhibit the tumor growth in vivo, and the underlying mechanism remains unclear. In this study, we demonstrated that azelnidipine could dual target SIRP $\alpha$ and PVR, simultaneously block the negative signal pathway, enhance the phagocytosis of tumor cells by macrophages, and significantly inhibit tumor growth. Moreover, azelnidipine could not only block the TIGIT/PVR pathway, but also reduce the expression of PVR, which may further enhance the anti-tumor effects. Our research may provide a potential mechanism for the tumor treatment by azelnidipine.

\section{Conclusions}

Here, we examined the over-expression of CD47 and PVR in a broad spectrum of cancers and tumor cell lines. We firstly discovered that an FDA-approved anti-hypotensive drug azelnidipine could be repositioned for cancer immunotherapy by dual targeting SIRP $\alpha$ and PVR through docking-based virtual screening. Azelnidipine could simultaneously bind to the key residues of SIRP $\alpha$ and PVR and block the interaction of both $\mathrm{CD} 47 / \mathrm{SIRP} \alpha$ and TIGIT/PVR. Azelnidipine could remarkably enhance the engulfment of tumor cells by macrophages in vitro. Irradiation could enhance the infiltration of MDSCs and upregulation of its expression of $\operatorname{SIRP} \alpha$, and azelnidipine alone or combined with irradiation could significantly inhibit the growth of MC38 tumors. Additionally, azelnidipine exerted anti-tumor effects by enhancing the quantity and activation of $\mathrm{CD} 8^{+} \mathrm{T}$ cells in the local tumor site, draining the lymph node and spleen. Our research provides a promising candidate for cancer immunotherapy, bridging the innate and adaptive immunity by a $\mathrm{CD} 47 / \mathrm{SIRP} \alpha$ and TIGIT/PVR blockade. 
Supplementary Materials: The following are available online at https:/ / www.mdpi.com/article/10 .3390/biom11050706/s1, Figure S1: The blocking efficacy of PD-1/PD-L1 interaction by azelnidipine. Figure S2: The antitumor effects of azelnidipine in $\mathrm{CD}^{+} \mathrm{T}$ cell depleted CT26 tumor model. Figure S3. The effects of azelnidipine on the expression of CD47 and PVR on CT26 tumor cells. Table S1: Candidate compounds through virtual screening.

Author Contributions: Conceptualization, X.Z., Y.G., and H.W.; methodology, X.Z. and H.W.; software, W.Z. (Wenshan Zhao); validation, W.Z. (Wenjie Zhai), L.Q., and Y.W.; formal analysis, X.Z. and H.W.; investigation, X.Z., H.W., L.J., Y.Q., Q.D., Y.S., and W.V.Z.; data curation, X.Z., H.W., and Y.G.; writing-original draft preparation, X.Z. and H.W.; writing-review and editing, H.W., W.Z. (Wenshan Zhao), and Y.G.; visualization, X.Z. and W.Z. (Wenshan Zhao); supervision, W.V.Z. and J.C.; project administration, Y.G., W.V.Z., and J.C.; funding acquisition, X.Z., W.Z. (Wenshan Zhao), Y.G., and J.C. All authors have read and agreed to the published version of the manuscript.

Funding: This research was funded by the National Natural Science Foundation of China (Nos. U20A20369 and 81822043 for Y.G.; Nos. 82002575 for X.Z. and Nos. 31700677 for W.S.Z.), Shenzhen Science and Technology Program (KQTD20190929173853397) and "Pearl River Talent Plan" Innovation and Entrepreneurship Team Project of Guangdong Province (2019ZT08Y464), Sanming Project of Medicine in Shenzhen (SZSM201612071), Shenzhen Key Medical Discipline Construction Fund (SZXK078), the China Postdoctoral Science Foundation (2020M672755) and the Cell Technology Center and Transformation Base, Innovation Center of Guangdong-Hong Kong-Macao Greater Bay Area, Ministry of Science and Technology of China (YCZYPT [2018]03-1).

Institutional Review Board Statement: The study was conducted according to the guidelines of the Declaration of Helsinki, and approved by the Ethics Committee of Zhengzhou University (ZZU20180312).

Informed Consent Statement: Not applicable.

Data Availability Statement: The datasets generated during and/or analyzed during the current study are available from the corresponding author on reasonable request.

Acknowledgments: Not applicable.

Conflicts of Interest: The authors declare that they have no competing interests.

\section{References}

1. Koyama, S.; Akbay, E.A.; Li, Y.Y.; Herter-Sprie, G.S.; Buczkowski, K.A.; Richards, W.G.; Gandhi, L.; Redig, A.J.; Rodig, S.J.; Asahina, H.; et al. Adaptive resistance to therapeutic PD-1 blockade is associated with upregulation of alternative immune checkpoints. Nat. Commun. 2016, 7, 10501. [CrossRef]

2. Zhou, X.; Zuo, C.; Li, W.; Shi, W.; Zhou, X.; Wang, H.; Chen, S.; Du, J.; Chen, G.; Zhai, W.; et al. A novel D peptide identified by mirror-image phage display blocks TIGIT/PVR for cancer immunotherapy. Angew. Chem. (Int. Ed. Engl.) 2020. [CrossRef]

3. Zhang, Q.; Bi, J.; Zheng, X.; Chen, Y.; Wang, H.; Wu, W.; Wang, Z.; Wu, Q.; Peng, H.; Wei, H.; et al. Blockade of the checkpoint receptor TIGIT prevents NK cell exhaustion and elicits potent anti-tumor immunity. Nat. Immunol. 2018, 19, 723-732. [CrossRef]

4. Bowers, J.R.; Readler, J.M.; Sharma, P.; Excoffon, K. Poliovirus receptor: More than a simple viral receptor. Virus Res. 2017, 242, 1-6. [CrossRef]

5. O'Donnell, J.S.; Madore, J.; Li, X.Y.; Smyth, M.J. Tumor intrinsic and extrinsic immune functions of CD155. Semin. Cancer Biol. 2020, 65, 189-196. [CrossRef]

6. Stengel, K.F.; Harden-Bowles, K.; Yu, X.; Rouge, L.; Yin, J.P.; Comps-Agrar, L.; Wiesmann, C.; Bazan, J.F.; Eaton, D.L.; Grogan, J.L. Structure of TIGIT immunoreceptor bound to poliovirus receptor reveals a cell-cell adhesion and signaling mechanism that requires cis-trans receptor clustering. Proc. Natl. Acad. Sci. USA 2012, 109, 5399-5404. [CrossRef]

7. Deuss, F.A.; Watson, G.M.; Fu, Z.; Rossjohn, J.; Berry, R. Structural basis for CD96 immune receptor recognition of Nectin-like protein-5, CD155. Structure 2019, 27, 219-228.e213. [CrossRef]

8. Inozume, T.; Yaguchi, T.; Furuta, J.; Harada, K.; Kawakami, Y.; Shimada, S. Melanoma cells control antimelanoma CTL responses via interaction between TIGIT and CD155 in the effector phase. J. Investig. Dermatol. 2016, 136, 255-263. [CrossRef]

9. Wu, L.; Mao, L.; Liu, J.F.; Chen, L.; Yu, G.T.; Yang, L.L.; Wu, H.; Bu, L.L.; Kulkarni, A.B.; Zhang, W.F.; et al. Blockade of TIGIT/CD155 signaling reverses T-cell exhaustion and enhances antitumor capability in head and neck squamous cell carcinoma. Cancer Immunol. Res. 2019, 7, 1700-1713. [CrossRef]

10. Zheng, Q.; Wang, B.; Gao, J.; Xin, N.; Wang, W.; Song, X.; Shao, Y.; Zhao, C. CD155 knockdown promotes apoptosis via AKT/Bcl-2/Bax in colon cancer cells. J. Cell. Mol. Med. 2018, 22, 131-140. [CrossRef]

11. Johnston, R.J.; Comps-Agrar, L.; Hackney, J.; Yu, X.; Huseni, M.; Yang, Y.; Park, S.; Javinal, V.; Chiu, H.; Irving, B.; et al. The immunoreceptor TIGIT regulates antitumor and antiviral CD8 ${ }^{(+)} \mathrm{T}$ cell effector function. Cancer Cell 2014, 26, 923-937. [CrossRef] 
12. Zhou, X.; Du, J.; Wang, H.; Chen, C.; Jiao, L.; Cheng, X.; Zhou, X.; Chen, S.; Gou, S.; Zhao, W.; et al. Repositioning liothyronine for cancer immunotherapy by blocking the interaction of immune checkpoint TIGIT/PVR. Cell Commun. Signal. CCS 2020, 18, 142. [CrossRef]

13. Galon, J.; Bruni, D. Approaches to treat immune hot, altered and cold tumours with combination immunotherapies. Nat. Rev. Drug Discov. 2019, 18, 197-218. [CrossRef]

14. Noy, R.; Pollard, J.W. Tumor-associated macrophages: From mechanisms to therapy. Immunity 2014, 41, 49-61. [CrossRef]

15. Li, X.; Liu, R.; Su, X.; Pan, Y.; Han, X.; Shao, C.; Shi, Y. Harnessing tumor-associated macrophages as aids for cancer immunotherapy. Mol. Cancer 2019, 18, 177. [CrossRef]

16. Rothlin, C.V.; Ghosh, S. Lifting the innate immune barriers to antitumor immunity. J. Immuno Ther. Cancer 2020, 8. [CrossRef]

17. Kuo, T.C.; Chen, A.; Harrabi, O.; Sockolosky, J.T.; Zhang, A.; Sangalang, E.; Doyle, L.V.; Kauder, S.E.; Fontaine, D.; Bollini, S.; et al. Targeting the myeloid checkpoint receptor SIRPalpha potentiates innate and adaptive immune responses to promote anti-tumor activity. J. Hematol. Oncol. 2020, 13, 160. [CrossRef]

18. Liu, X.; Liu, L.; Ren, Z.; Yang, K.; Xu, H.; Luan, Y.; Fu, K.; Guo, J.; Peng, H.; Zhu, M.; et al. Dual targeting of innate and adaptive checkpoints on tumor cells limits immune evasion. Cell Rep. 2018, 24, 2101-2111. [CrossRef]

19. Liu, B.; Guo, H.; Xu, J.; Qin, T.; Guo, Q.; Gu, N.; Zhang, D.; Qian, W.; Dai, J.; Hou, S.; et al. Elimination of tumor by CD47/PD-L1 dual-targeting fusion protein that engages innate and adaptive immune responses. $m A b s$ 2018, 10, 315-324. [CrossRef]

20. Ashburn, T.T.; Thor, K.B. Drug repositioning: Identifying and developing new uses for existing drugs. Nat. Rev. Drug Discov. 2004, 3, 673-683. [CrossRef]

21. Wurth, R.; Thellung, S.; Bajetto, A.; Mazzanti, M.; Florio, T.; Barbieri, F. Drug-repositioning opportunities for cancer therapy: Novel molecular targets for known compounds. Drug Discov. Today 2016, 21, 190-199. [CrossRef] [PubMed]

22. Pushpakom, S.; Iorio, F.; Eyers, P.A.; Escott, K.J.; Hopper, S.; Wells, A.; Doig, A.; Guilliams, T.; Latimer, J.; McNamee, C.; et al Drug repurposing: Progress, challenges and recommendations. Nat. Rev. Drug Discov. 2019, 18, 41-58. [CrossRef] [PubMed]

23. Li, H.; Liu, A.; Zhao, Z.; Xu, Y.; Lin, J.; Jou, D.; Li, C. Fragment-based drug design and drug repositioning using multiple ligand simultaneous docking (MLSD): Identifying celecoxib and template compounds as novel inhibitors of signal transducer and activator of transcription 3 (STAT3). J. Med. Chem. 2011, 54, 5592-5596. [CrossRef]

24. Kim, K.; Yang, W.-H.; Jung, Y.-S.; Cha, J.-h. A new aspect of an old friend: The beneficial effect of metformin on anti-tumor immunity. BMB Rep. 2020, 53, 512-520. [CrossRef] [PubMed]

25. Li, L.; Wang, L.; Li, J.; Fan, Z.; Yang, L.; Zhang, Z.; Zhang, C.; Yue, D.; Qin, G.; Zhang, T.; et al. Metformin-Induced Reduction of CD39 and CD73 Blocks Myeloid-Derived Suppressor Cell Activity in Patients with Ovarian Cancer. Cancer Res. 2018, 78, 1779-1791. [CrossRef] [PubMed]

26. Cha, J.H.; Yang, W.H.; Xia, W.; Wei, Y.; Chan, L.C.; Lim, S.O.; Li, C.W.; Kim, T.; Chang, S.S.; Lee, H.H.; et al. Metformin Promotes Antitumor Immunity via Endoplasmic-Reticulum-Associated Degradation of PD-L1. Mol. Cell 2018, 71, 606-620.e607. [CrossRef] [PubMed]

27. Luo, F.; Luo, M.; Rong, Q.X.; Zhang, H.; Chen, Z.; Wang, F.; Zhao, H.Y.; Fu, L.W. Niclosamide, an antihelmintic drug, enhances efficacy of PD-1/PD-L1 immune checkpoint blockade in non-small cell lung cancer. J Immuno Ther. Cancer 2019, 7, 245. [CrossRef]

28. Hatherley, D.; Harlos, K.; Dunlop, D.C.; Stuart, D.I.; Barclay, A.N. The structure of the macrophage signal regulatory protein alpha (SIRPalpha) inhibitory receptor reveals a binding face reminiscent of that used by T cell receptors. J. Biol. Chem. 2007, 282, 14567-14575. [CrossRef]

29. Zhai, W.; Zhou, X.; Du, J.; Gao, Y. In vitro assay for the development of small molecule inhibitors targeting PD-1/PD-L1. In Methods in Enzymology; Academic Press: Cambridge, MA, USA, 2019. [CrossRef]

30. Zhai, W.; Zhou, X.; Wang, H.; Li, W.; Chen, G.; Sui, X.; Li, G.; Qi, Y.; Gao, Y. A novel cyclic peptide targeting LAG-3 for cancer immunotherapy by activating antigen-specific CD8 ${ }^{(+)} \mathrm{T}$ cell responses. Acta Pharm. Sin. B 2020, 10, 1047-1060. [CrossRef] [PubMed]

31. Logtenberg, M.E.W.; Scheeren, F.A.; Schumacher, T.N. The CD47-SIRPalpha immune checkpoint. Immunity 2020, 52, 742-752. [CrossRef]

32. Burugu, S.; Dancsok, A.R.; Nielsen, T.O. Emerging targets in cancer immunotherapy. Semin. Cancer Biol. 2017. [CrossRef] [PubMed]

33. Li, T.; Fan, J.; Wang, B.; Traugh, N.; Chen, Q.; Liu, J.S.; Li, B.; Liu, X.S. TIMER: A web server for comprehensive analysis of tumor-infiltrating immune cells. Cancer Res. 2017, 77, e108-e110. [CrossRef]

34. Candas-Green, D.; Xie, B.; Huang, J.; Fan, M.; Wang, A.; Menaa, C.; Zhang, Y.; Zhang, L.; Jing, D.; Azghadi, S.; et al. Dual blockade of CD47 and HER2 eliminates radioresistant breast cancer cells. Nat. Commun. 2020, 11, 4591. [CrossRef]

35. Pai, S.; Bamodu, O.A.; Lin, Y.K.; Lin, C.S.; Chu, P.Y.; Chien, M.H.; Wang, L.S.; Hsiao, M.; Yeh, C.T.; Tsai, J.T. CD47-SIRPalpha signaling induces epithelial-mesenchymal transition and cancer stemness and links to a poor prognosis in patients with oral squamous cell carcinoma. Cells 2019, 8, 1658. [CrossRef]

36. Kerr, W.G.; Chisholm, J.D. The next generation of immunotherapy for cancer: Small molecules could make big waves. J. Immunol. 2019, 202, 11-19. [CrossRef]

37. Huck, B.R.; Kötzner, L.; Urbahns, K. Small molecules drive big improvements in immuno-oncology therapies. Angew. Chem. (Int. Ed. Engl.) 2018, 57, 4412-4428. [CrossRef] [PubMed] 
38. Hatherley, D.; Graham, S.C.; Turner, J.; Harlos, K.; Stuart, D.I.; Barclay, A.N. Paired receptor specificity explained by structures of signal regulatory proteins alone and complexed with CD47. Mol. Cell 2008, 31, 266-277. [CrossRef]

39. Advani, R.; Flinn, I.; Popplewell, L.; Forero, A.; Bartlett, N.L.; Ghosh, N.; Kline, J.; Roschewski, M.; LaCasce, A.; Collins, G.P.; et al. CD47 blockade by Hu5F9-G4 and Rituximab in Non-Hodgkin's Lymphoma. N. Engl. J. Med. 2018, 379, 1711-1721. [CrossRef]

40. Zhou, X.; Du, J.; Zhou, X.; Niu, X.; Li, W.; Chen, C.; Lv, S.; Wu, A.; Gou, S.; Sun, Y.; et al. Computer-aided design of PVR mutants with enhanced binding affinity to TIGIT. Cell Commun. Signal. CCS 2021, 19, 12. [CrossRef]

41. Deng, L.; Liang, H.; Burnette, B.; Beckett, M.; Darga, T.; Weichselbaum, R.R.; Fu, Y.X. Irradiation and anti-PD-L1 treatment synergistically promote antitumor immunity in mice. J. Clin. Investig. 2014, 124, 687-695. [CrossRef]

42. Bronte, V.; Brandau, S.; Chen, S.H.; Colombo, M.P.; Frey, A.B.; Greten, T.F.; Mandruzzato, S.; Murray, P.J.; Ochoa, A.; OstrandRosenberg, S.; et al. Recommendations for myeloid-derived suppressor cell nomenclature and characterization standards. Nat. Commun. 2016, 7, 12150. [CrossRef]

43. Wang, H.; Sun, Y.; Zhou, X.; Chen, C.; Jiao, L.; Li, W.; Gou, S.; Li, Y.; Du, J.; Chen, G.; et al. CD47/SIRP $\alpha$ blocking peptide identification and synergistic effect with irradiation for cancer immunotherapy. J. Immuno Ther. Cancer 2020, 8, e000905. [CrossRef]

44. Liu, X.; Pu, Y.; Cron, K.; Deng, L.; Kline, J.; Frazier, W.A.; Xu, H.; Peng, H.; Fu, Y.X.; Xu, M.M. CD47 blockade triggers T cell-mediated destruction of immunogenic tumors. Nat. Med. 2015, 21, 1209-1215. [CrossRef] [PubMed]

45. Hazama, D.; Yin, Y.; Murata, Y.; Matsuda, M.; Okamoto, T.; Tanaka, D.; Terasaka, N.; Zhao, J.; Sakamoto, M.; Kakuchi, Y.; et al. Macrocyclic peptide-mediated blockade of the CD47-SIRPalpha interaction as a potential cancer immunotherapy. Cell Chem. Biol. 2020, 27, 1181-1191.e1187. [CrossRef]

46. Sanchez-Correa, B.; Valhondo, I.; Hassouneh, F.; Lopez-Sejas, N.; Pera, A.; Bergua, J.M.; Arcos, M.J.; Banas, H.; Casas-Aviles, I.; Duran, E.; et al. DNAM-1 and the TIGIT/PVRIG/TACTILE axis: Novel immune checkpoints for natural killer cell-based cancer immunotherapy. Cancers 2019, 11, 877. [CrossRef]

47. Anighoro, A.; Bajorath, J.; Rastelli, G. Polypharmacology: Challenges and opportunities in drug discovery. J. Med. Chem. 2014, 57, 7874-7887. [CrossRef]

48. Birx, D.L.; Berger, M.; Fleisher, T.A. The interference of T cell activation by calcium channel blocking agents. J. Immunol. 1984, 133, 2904-2909.

49. Wright, B.; Zeidman, I.; Greig, R.; Poste, G. Inhibition of macrophage activation by calcium channel blockers and calmodulin antagonists. Cell. Immunol. 1985, 95, 46-53. [CrossRef]

50. Liu, W.; Matsumori, A. Calcium channel blockers and modulation of innate immunity. Curr. Opin. Infect. Dis. 2011, 24, 254-258. [CrossRef]

51. Matsumori, A.; Nishio, R.; Nose, Y. Calcium channel blockers differentially modulate cytokine production by peripheral blood mononuclear cells. Circ. J. Off. J. Jpn. Circ. Soc. 2010, 74, 567-571. [CrossRef]

52. Komoda, H.; Shiraki, A.; Oyama, J.I.; Nishikido, T.; Node, K. Azelnidipine inhibits the differentiation and activation of THP-1 macrophages through the L-type calcium channel. J. Atheroscler. Thromb. 2018, 25, 690-697. [CrossRef]

53. Miura, R.; Nakamura, K.; Miura, D.; Miura, A.; Kajiya, M.; Hisamatsu, K.; Nagase, S.; Morita, H.; Kusano, K.F.; Matsubara, H.; et al. Cytokine reducing effect of azelnidipine in human peripheral blood mononuclear cells. Biol. Pharm. Bull. 2010, 33, 1148-1151. [CrossRef] [PubMed]

54. Abe, T.; Fuse, I.; Narita, M.; Takahashi, M.; Aizawa, Y. Combination use of immune complexes and a Ca ${ }^{2(+)}$ channel blocker azelnidipine enhances interleukin-12 p40 secretion without $\mathrm{T}$ helper type 17 cytokine secretion in human monocyte-derived dendritic cells. Clin. Exp. Immunol. 2009, 156, 405-412. [CrossRef] [PubMed]

55. Chiu-Hung, C.; Show-Mei, C.; Tzong-Der, W.; Nai-Wan, H. Use of Azelnidipine in Preaparing Medicinal Composition for Treating Cancers. Patent EP3222278(A4), 22 October 2015.

56. Chiu-Hung, C.; Show-Mei, C.; Tzong-Der, W.; Nai-Wan, H. New Indication of Azelnidipine Pharmaceutical Composition for Treating Cancer. Patent US2017312260(A1), 22 October 2015. 